\title{
Androgen receptor activity promotes resistance to BRAF-targeted melanoma therapy
}

Jennifer Wargo ( $\sim$ jwargo@mdanderson.org )

MD Anderson https://orcid.org/0000-0003-3438-7576

\section{Christopher Vellano}

The University of Texas MD Anderson Cancer Center

\section{Michael White}

The University of Texas MD Anderson Cancer Center

\section{Miles Andrews}

Monash University https://orcid.org/0000-0003-1231-8641

\section{Joseph Daniele}

The University of Texas MD Anderson Cancer Center

\section{Mark Titus}

The University of Texas MD Anderson Cancer Center

\section{Gabriel Ologun}

The University of Texas MD Anderson Cancer Center

\section{Alex Cogdill}

MD Anderson Cancer Center, Department of Surgical Oncology

\section{Golnaz Morad}

The University of Texas MD Anderson Cancer Center https://orcid.org/0000-0002-9460-618X

\section{Jennifer McQuade}

Department of Melanoma Medical Oncology, The University of Texas MD Anderson Cancer Center

\section{Rohit Thakur}

The University of Texas MD Anderson Cancer Center

\section{Peter Prieto}

University of Rochester Medical Center

\section{Alexander Lazar}

The University of Texas MD Anderson Cancer Center https://orcid.org/0000-0002-6395-4499

\section{Md Abdul Wadud Khan}

University of Texas MD Anderson Cancer Center

\section{Beth Helmink}

The University of Texas MD Anderson Cancer Center

\section{Michael Davies}

MD Anderson https://orcid.org/0000-0002-0977-0912 
University of Texas M.D. Anderson Cancer Center https://orcid.org/0000-0001-6876-278X Jeffrey Kovacs

The University of Texas MD Anderson Cancer Center

\section{Scott Woodman}

The University of Texas MD Anderson Cancer Center

\section{Hussein Tawbi}

University of Texas MD Anderson Cancer Center https://orcid.org/0000-0003-1942-851X

\section{Sapna Patel}

MD Anderson Cancer Center

\section{Patrick Hwu}

The University of Texas MD Anderson Cancer Center

\section{Michael Peoples}

University of Texas MD Anderson Cancer Center

\section{Jeffrey Lee}

MD Anderson Cancer Center, Department of Surgical Oncology

\section{Zachary Cooper}

AstraZeneca https://orcid.org/0000-0003-1059-0940

\section{Haifeng Zhu}

The University of Texas MD Anderson Cancer Center

\section{Guang Gao}

University of Texas MD Anderson Cancer Center

Jeffrey Gershenwald

The University of Texas MD Anderson Cancer Center

\section{Anthony Lucci}

UT MD Anderson Cancer Center

\section{Emily Keung}

University of Texas MD Anderson Cancer Center

\section{Merrick Ross}

The University of Texas MD Anderson Cancer Center

\section{Khalida Wani}

M. D. Anderson Cancer Center

\section{Michael Tetzlaff}

MD Anderson

\section{Lauren Haydu}

The University of Texas MD Anderson Cancer Center

\section{Mikhila Mahendra}

University of Texas MD Anderson Cancer Center

\section{XioYan Ma}

The University of Texas MD Anderson Cancer Center 


\section{Christopher Logothetis}

The University of Texas MD Anderson Cancer Center

\section{Zachary Kulstad}

The University of Texas MD Anderson Cancer Center

\section{Sarah Johnson}

University of Texas MD Anderson Cancer Center

\section{Courtney Hudgens}

The University of Texas MD Anderson Cancer Center https://orcid.org/0000-0001-8312-7485

\section{Ningping Feng}

University of Texas MD Anderson Cancer Center

\section{Lorenzo Federico}

The University of Texas MD Anderson Cancer Center

\section{Elizabeth Burton}

MD Anderson Cancer Center, Department of Surgical Oncology

\section{Swathi Arur}

The University of Texas MD Anderson Cancer Center https://orcid.org/0000-0002-6941-2711

\section{Timothy Heffernan}

The University of Texas MD Anderson Cancer Center

\section{Joseph Marszalek}

University of Texas MD Anderson Cancer Center

\section{Research Article}

Keywords: cancer, targeted therapy, androgen, BRAF

Posted Date: August 17th, 2020

DOI: https://doi.org/10.21203/rs.3.rs-58480/v1

License: (c) (1) This work is licensed under a Creative Commons Attribution 4.0 International License. Read Full License

Version of Record: A version of this preprint was published at Nature on June 15th, 2022. See the published version at https://doi.org/10.1038/s41586-022-04833-8. 


\section{Abstract}

Treatment with molecularly-targeted therapy has revolutionized cancer care, including BRAF/MEKtargeted melanoma therapy. However responses are heterogenous and frequently not long-lasting. Novel strategies to target resistance are needed. We studied a cohort of patients with resectable metastatic melanoma treated with neoadjuvant BRAF/MEK-targeted therapy $(n=52)$ and noted a strong sexual dimorphism in response to treatment, with female patients demonstrating significantly higher rates of a major pathologic response (MPR) ( $p=0.0001)$. RNA sequencing of tumors demonstrated enrichment of androgen-related genes in those failing to achieve MPR. Pre-clinical studies validated these findings, with significantly increased tumor growth in male vs female mice treated with BRAF/MEK inhibitors (BRAF/MEKi) ( $p=0.0005)$. Androgen receptor $(A R)$ expression was upregulated in tumors of BRAF/MEKitreated mice, and modulation of AR signaling via AR-blockade or castration was associated with significantly slower tumor growth $(p=0.0001$ and $p=0.00004$, respectively). Together, these results have important implications in the context of treatment with BRAF/MEKi-targeted therapy.

\section{Main Text}

To date, multiple studies have demonstrated that male sex is associated with worse outcomes in patients with melanoma, including patients with stage III and IV disease treated with targeted therapy ${ }^{1-3}$. While these differences have been noted in melanoma as well as other malignancies, the driving factors are incompletely understood ${ }^{4-8}$. Variations in the immune background ${ }^{9,10}$, tumor microenvironment ${ }^{11-14}$ and tumor cell susceptibility to targeted therapy ${ }^{9,15}$ have been posited as driving factors of this sexual dimorphism in treatment outcomes. Additionally, innate hormonal differences in the estrogen and androgen pathways have been suggested as potential mechanisms driving observed disparities in melanoma preclinical models ${ }^{12,13,16-18}$ of immune checkpoint blockade ${ }^{19,20}$ and targeted therapy ${ }^{21}$. However further insights from patient cohort and pre-clinical studies are needed to help derive rational combinatorial strategies to improve patient survival.

We and others have recently shown neoadjuvant targeted therapy to be of significant clinical benefit in patients with high-risk, surgically resectable locoregional metastatic melanoma ${ }^{22,23}$. These studies provide a unique opportunity for in-depth molecular characterization and longitudinal study of biospecimens in a more homogeneous patient population. Futhermore, this work has demonstrated that early endpoints, including major pathologic response after neoadjuvant chemotherapy correlate with long-term outcomes ${ }^{22,24}$. In one of the first trials performed, we noted a strong sexual dimorphism in response to treatment with neoadjuvant BRAF/MEK targeted therapy in patients with locoregional melanoma metastases ${ }^{22,25}$, though sample size was limited (Supplemental Figure 1A).

To build on these findings, we pooled data from a cohort of melanoma patients with locoregional metastases who were treated with neoadjuvant BRAF-MEK targeted therapy within and outside of clincial trials (Figure 1A, Supplementary Table 1). Patients were treated with neoadjuvant BRAF-MEK targeted therapy for a duration of 8-12 weeks prior to definitive surgical resection. Pathologic responses were 
assessed, and patients were dichotomized into those having a major pathologic response (MPR) $(\leq 10 \%$ viable tumor) versus those without a major pathologic response ( $>10 \%$ viable tumor) as defined by the International Neoadjuvant Melanoma Consortium ${ }^{26}$. In this pooled cohort, we again noted a strong sex difference in therapeutic response, with female patients experiencing a significantly higher rate of MPR on neoadjuvant dabrafenib and trametinib (DT) (22/30,66\%) compared to male patients $(3 / 22,14 \%)$ (odds ratio of $12.795 \% \mathrm{Cl}$ [3.0-53.2] $\mathrm{p}=0.001$ ) (Figure $1 \mathrm{~B}$, Supplementary Table 1). No other clinical factors were significantly associated with achievement of MPR on logistic regression analysis, including age $(p=0.70)$, stage IIIC or $D(p=0.45)$, stage IV $(p=0.53)$, BRAF V600 E versus V600 non-E mutation $(p=0.57), E C O G$ performance status $(p=0.94)$, body mass index $(B M I)(>30)(p=0.80)$, serum lactate dehydrogenase $(L D H)(p=0.27)$, or recurrent disease $(p=0.29)$. Within the female subset, there was no association between menopausal status (pre- vs post-) and MPR; 9/13 (69\%) pre-menopausal women and $11 / 17(65 \%)$ post-menopausal women achieved a MPR $(p=0.79)$. Females also had significantly improved progression-free survival as compared to male patients (Figure $1 \mathrm{C}, \mathrm{p}=0.043$ ).

To explore the potential molecular mechanisms underlying this observed sex-difference in response to neoadjuvant therapy, we analyzed the gene expression changes using available RNA sequencing analysis of pre-treatment samples of patient tumors from the initial clinical trial cohort ( $n=4$ female, 5 male) . From this analysis, we observed an enrichment of androgen-related pathways in non-responders, and estrogen-related pathways in responders (Figure 1D, Supplementary Figure 1B). We next assessed androgen receptor (AR) expression via immunohistochemical staining for androgen receptor percent positive nuclei in available matched pre- and on-treatment tumor biopsy specimens ( $n=4$ female, $n=4$ male). This identified a trend toward higher AR expression in tumors after treatment with BRAF-MEK targeted therapy in both male and female patients (Figure 1E, $p=0.06$, Supplemental Table 2), with all male patient tumors tested exhibiting increased expression post-treatment.

Intrigued by these results, we then functionally evaluated the impact of sexual dimorphism on response to neoadjuvant targeted therapy using the $\mathrm{Braf}^{\mathrm{V} 600 \mathrm{E}} / \mathrm{Pten}^{-/-}$(BP) syngeneic murine melanoma model (female genotype). To do this, C57BI/6 (immune competent) or CD-1 nude (immune incompetent) male and female mice were implanted subcutaneously with BP cells and treated daily with dabrafenib + trametinib (DT) once tumors reached an average size of $\sim 400-600 \mathrm{~mm}^{3}$ (Supplemental Fig. 3A). In these studies, DT treatment was associated with delayed tumor outgrowth in all mice compared to vehicletreated mice as expected. However male mice demonstrated significantly impaired tumor control on DT compared to female mice in an immunocompetent model as well as in nude mice $(p<0.0005$ and $p<$ 0.00001 respectively, Figure 2A, B and Supplemental Figure 3B, C), corroborating our findings in the clinical cohort, and suggesting that the mechanism is not immune-mediated.

Building upon the observation that AR staining was elevated is post-treatment samples samples of patients on neoadjuvant DT, we next analyzed AR expression via bulk transcriptome analysis and immunofluorescence in tumors of vehicle- and DT-treated mice with the hypothesis that DT treatment would be elevated in DT-treated mice. In these studies, a modest increase in AR mRNA expression was noted in females and males upon treatment with DT ( $p<0.005$ and $p=0.16$ respectively) (Figure $2 C$ ). 
However, AR protein expression was significantly increased in male DT-treated mice versus vehicle control $(p=0.0003$, Figure 2D, E), suggesting a potential role for testosterone in stabilizing AR and mediating resistance. Interestingly, there was no difference in AR protein expression in female DT-treated mice compared to vehicle control ( $p=0.9$, Figure $2 D, E)$. Reverse phase protein array (RPPA) of tumor samples increased phosphorylation (activation) of components of the MAPK and PI3K/mTOR pathways in male versus female mice following treatment with DT, which may also underlie differential response (Figure 2F).

Following this, we next sought to test whether AR signaling was underlying the observed treatment resistance. To do this, we evaluated the effect of modulating AR signaling on DT response through either AR blockade or castration in male mice, or via administration of testosterone in female mice (Supplementary Figure 3A,B). Results demonstrated significantly increased tumor outgrowth in female mice receiving DT + testosterone versus DT alone (Figure $3 A ; p<0.00001$, Supplementary Figure $3 C$ ). Conversely, male mice treated with DT + AR blockade (enzalutamide) or castration demonstrated delayed tumor outgrowth compared to male mice treated with DT alone (Figure 3B; $p=0.0001$ and $p=0.00004$, respectively, Supplementary Figure 3D,E). Notably, treatment with DT + testosterone in males was associated with increased tumor growth over DT alone (Figure $3 B ; p=0.0005$ ), suggesting that testosterone-regulated activity of AR contributes to the differential response to DT. Treatment with exogenous testosterone reversed the protective effects of both castration and AR blockade with enzalutamide in male mice (Figure $3 C$ and 3D; $p<0.00001$ and $p=0.005$, respectively). Teatment with AR blockade + DT in female mice was associated with delayed tumor outgrowth compared to DT treatment alone (Figure $3 E ; p=0.007$ ) suggesting that this combination may also be effective in females. Similar to males, treatment with exogenous testosterone + DT and AR blockade tended to abrogate the effects of AR blockade on DT treatment ( $p=0.08)$. (Figure 3E; $p=0.02)$.

We next assessed levels of testosterone and AR expression in tumors of male and female mice treated with DT with or without modulation of AR signaling (via AR blockade, castration, or exogenous testosterone administration). In these studies, treatment with strategies to reduce AR signaling in male mice (castration, enzalutamide) were associated with reduced intra-tumoral testosterone levels (Figure 3F; 138.7 and 4.6 fold decrease, respectively). Conversely, strategies to increase AR signaling (exogenous testosterone) in female mice was associated with increased intra-tumoral testosterone levels (Figure 3G; 263.5 fold increase). Androgen receptor staining mirrored these findings, with a trend for decreased AR expression in DT + enzalutamide and a significant reduction in the DT + castration groups versus DT alone in male mice, which was abrogated with administration of exogenous testosterone (Figure $3 \mathrm{H}$ and $3 l, p=0.3$ for $D T+$ enzalutamide, $p=0.0003$ for $D T+$ castration). Although there is a slight increase in the percentage of AR-positive cells following DT treatment, expression remains very low. While a trend toward an increase in AR positive nuclei in mice receiving DT + testosterone was noted as well (Figure $\mathbf{3 H}$ and $3 \mathrm{l}, \mathrm{p}=0.04, \mathrm{p}=0.06$ respecitvely, Supplemental Figure 3F).

To validate that androgen receptor signaling was driving resistance to BRAF-MEK targeted therapy, we 
next generated AR knockout melanoma cells using CRISPR technology and implanted these into male and female syngeneic mice. These studies demonstrated that the modulation of response to DT treatment via administration of testosterone or enzalutamide was lost (Figure $\mathbf{3} \mathbf{J}$ and $\mathbf{3 K}$ ), suggesting that the phenotypes observed in our prior studies are directly related to androgen receptor signaling. Negligible AR expression was confirmed via immunofluorescence (Supplementary Figure 3G). Taken together, these data demonstrate that AR stabilization by testosterone contributes to resistance to BRAFMEK targeted melanoma therapy, and highlights the potential for AR blockade by enzalutamide as a therapeutic strategy to overcome this resistance. Though our current studies only explored these relationships in melanoma, studies interrogating AR signaling in the context of MAPK-targeted therapy are warranted across cancer types.

\section{Methods}

\section{Clinical cohorts:}

Patients enrolled in the initial clinical trial cohort were as previously described ${ }^{1}$. Briefly, patients $\geq 18$ years old with histologically-proven clinical stage III or oligometastatic stage IV BRAF V600E/K melanoma deemed resectable by multidisciplinary consensus and measurable disease by RECIST 1.1 criteria were enrolled and those randomized to the experimental arm received 8 weeks of neoadjuvant dabrafenib (150mg PO BD) plus trametinib (2mg PO daily) prior to surgical resection, followed by up to 44 weeks of adjuvant dabrafenib and trametinib (neoDT) $(n=12)$. Additional patients $(n=20)$ from a single-arm study of neoDT along with an additional retrospective cohort of patients treated off protocol for logistic reasons with neoDT $(n=16)$, Dabrafenib $(n=3)$, and Encorafenib plus Binimetinib $(n=1)$ were included. Radiographic responses to neoadjuvant therapy were determined at week 8 prior to surgery and pathologic responses were determined by microscopic examination of the complete surgical specimen by a melanoma pathologist, including SOX10 immunostains when applicable to confirm presence or absence of viable melanoma cells.

\section{RNA sequencing:}

Tumor biopsies were obtained as feasible by punch or core biopsy prior to and during the neoadjuvant treatment period. Fresh-frozen tumor biopsy material was used for RNA sequencing library preparation. Total RNA was extracted from snap-frozen tumor specimens using the AllPrep DNA/RNA/miRNA Universal Kit (Qiagen) following assessment of tumor content by a Pathologist, and macrodissection of tumor bed if required. RNA quality was assessed on an Agilent 2100 Bioanalyzer using the Agilent RNA 6000 Nano Chip with smear analysis to determine DV200 and original RNA concentration. Based on RNA quality, 40-80ng of total RNA from each sample then underwent library preparation using the Illumina TruSeq RNA Access Library Prep kit according to the manufacturer's protocol. Barcoded libraries were pooled to produce final 10-12 plex pools prior to sequencing on an Illumina NextSeq 500 sequencer using one high-output run per pool of 76bp paired-end reads, generating 8 fastq files (4 lanes, paired reads) per sample. 
RNA-seq FASTQ files were first processed through FastQC $(\mathrm{v} 0.11 .5)^{2}$, a quality control tool to evaluate the quality of sequencing reads at both the base and read levels. Reads having ${ }^{3} 15$ contiguous low-quality bases (phred score <20) were removed from the FASTQ files prior to STAR 2-pass alignment $(v 2.5 .3)^{3}$ with default parameters to generate one BAM file for each sequencing event. After that, RNA-SeQC

(v1.1.8) ${ }^{4}$ was used to generate quality control metrics including read counts, coverage, and correlation. A matrix of Spearman correlation coefficients amongst all sequenced samples was subsequently generated by RNA-SeQC and after careful review the sequencing data generated from one library pool that showed poor correlation with other library pools from the same RNA sample was removed before sample-level merging of BAM files.

HTSeq-count (v0.9.1) ${ }^{5}$ tool was applied to aligned RNA-seq BAM files to count how many aligned reads overlapped with the exons of each gene. The raw read counts generated from HTSeq-count were normalized into fragments per kilobase of transcript per million mapped reads (FPKM) using the RNA-seq quantification approach suggested by the bioinformatics team of NCl Genomic Data Commons (GDC) [https://docs.gdc.cancer.gov/Data/Bioinformatics_Pipelines/Expression_mRNA_Pipeline/]. Briefly, FPKM normalizes read count by dividing it by the gene length and the total number of reads mapped to proteincoding genes using a calculation described below:

$$
F P K M=\frac{R C_{g} * 10^{9}}{R C_{p c} * L}
$$

$\mathrm{RC}_{\mathrm{g}}$, number of reads mapped to the gene; $\mathrm{RC}_{\mathrm{pc}}$ : number of reads mapped to all protein-coding genes; $\mathrm{L}$, length of the gene in base pairs (calculated as the sum of all exons in a gene). The FPKM values were then log2-transformed for further downstream analyses.

\section{RNA sequencing differential expression and gene set enrichment analysis:}

The HTSeq normalized read count data for all expressed coding transcripts were processed by DESeq2 $(v 3.6)^{6}$ software to identify differentially expressed genes (DEGs) between two response (responder vs. non-responder) groups. A cut-off for gene expression fold change of $\geq 2$ or $\leq-0.5$ and a FDR (q-value) of $<.05$ was applied to select the most differentially expressed genes. Gene set enrichment analyses were performed using the GSEA software developed at the Broad Institute, using vst-normalised ${ }^{7}$ input transcriptome expression data and querying the MSigDB Hallmark gene sets with default parameters.

\section{Animals and xenograft studies:}

Female or male C57BL/ 6 (strain code: 0000664, purchased from Jackson Lab), aged 6 to 12 weeks, and weighing approximately 20 to $25 \mathrm{~g}$ were used for in vivo studies. Female or male CD-1 nude mice (strain code: 086, purchased from Charles River Laboratories), aged 6 to 12 weeks, and weighing approximately 
25-40 $\mathrm{g}$ were used for the immunodeficient model in vivo studies. Animal health was monitored daily by observation and sentinel blood sample analysis. Animal experiments were conducted in accordance with the Guideline of IACUC at MDACC.

BP cells were scaled up in DMEM culture media supplemented with $10 \% \mathrm{FBS}$, harvested, and prepared so that each mouse received $0.8 \times 10^{6}$ cells in $0.2 \mathrm{~mL}$ PBS. Cells were implanted subcutaneously in the right flank of each mouse. For some male mice, physical castration was required and performed two weeks before treatment or before cell implantation. Testosterone pellets (Innovative Research of America) were implanted subcutaneously in the left flank one week before treatment. Trametinib (Chemietek) at 1 $\mathrm{mg} / \mathrm{kg}$ and Dabrafenib (Chemietek) at $30 \mathrm{mg} / \mathrm{kg}$ were suspended at concentrations as needed in an aqueous vehicle containing $0.5 \%$ Hydroxypropylmethylcellulose and $0.2 \%$ Tween 80 in distilled water and adjusted to $\mathrm{pH} 8.80$ with diluted $\mathrm{NaOH}$ solution. Enzalutamide (Chemietek) at $10 \mathrm{mg} / \mathrm{kg}$ was suspended in $1 \%$ carboxymethyl cellulose (Sigma), 0.1\% Tween-80, 5\% DMSO.

BP tumors were monitored by caliper before randomly sorting and dividing into experimental groups ( $n=10$ mice per group). Treatment was started from day 14 to 17 days post-implantation depending on mouse strain and gender. Vehicle controls, mixture of Trametinib at $1 \mathrm{mg} / \mathrm{kg}$ and Dabrafenib at 30 $\mathrm{mg} / \mathrm{kg}$ or Enzalutamide at $10 \mathrm{mg} / \mathrm{kg}$ were given orally using a sterile 1- $\mathrm{mL}$ syringe and 18-gavage needle for 21 days. Dosing was 5 hours apart between administration of Trametinib + Dabrafenib and Enzalutamide for these specific treatment groups.

Tumor volume was calculated using the following formula: $\left[\mathrm{LX}\left(\mathrm{W}^{2}\right) / 2\right]$ (in which $L+$ length of tumors; W =width of tumor). Tumor and plasma were harvested 4 hours after the last dose. Tumors were snap of frozen and the plasma was divided for monitoring drug concentrations and hormone levels. Endpoint samples were collected and processed for gene expression using Quant-seq and protein expression via Reverse Phase Protein Array (RPPA). RPPA analysis was performed the MD Anderson Cancer Center's functional proteomic RPPA core facility as previously described ${ }^{8}$.

\section{Quant-seq library construction and sequencing.}

$1000 \mathrm{ng}$ or $500 \mathrm{ng}$ of DNAse-treated RNA samples were converted to cDNA using a QuantSeq 3' mRNA according to the manufacturer's protocol (Lexogen, Vienna, Austria). The libraries were amplified with 12 or 13 PCR Cycles and purified using the provided Lexogen. The purified libraries were quantified using a Kapa library quantification kit (KAPA biosystems) and loaded on NextSeq 500 Sequencer (Illumina, San Diego, CA) at a final concentration of $2.6 \mathrm{pM}$ to perform cluster generation, followed by $1 \times 76 \mathrm{bp}$ sequencing on NextSeq 500 Sequencer (Illumina).

\section{Androgen Receptor CRISPR KO in BP cell lines:}

Three guide sequences were designed to target a 100 bp region of Exon 1 using HorizonDiscovery's CRISPR Design Tool. Guide 1 (gacttgggtagtctacatgg AGG) was cloned into pLentiCRISPR.v2 according to 
addgene lentiCRISPRv2 and lentiGuide oligo cloning protocol for the purpose of pool selection. Guide 2 (gcttgatacgggcgtgtggat GGG) and Guide 3 (ctggagaacccattggacta CGG) were ordered as crRNA's (IDT).

The Neon electroporation system was used to transfect ribonucleoprotein (RNP) complex + plasmid into the BP cells. The crRNAs were rehydrated to $200 \mathrm{nM}$ and pooled in equal volume ( $3 \mu \mathrm{L}$ each) for annealing with Alt-R CRISPR-Cas 9 tracrRNA-ATTO 550 (IDT; 1075928); $95^{\circ} \mathrm{C} \times 5 \mathrm{~min}$, slowly cool to $10^{\circ} \mathrm{C}$ at $0.1 \mathrm{C} / \mathrm{sec}$. $5 \mu \mathrm{L}$ of crRNA:tracrRNA were combined with $5 \mu \mathrm{L}$ of Alt-R S.p. HiFi Cas 9 nuclease (IDT; 1081060) at room temperature for $10 \mathrm{~min}$ to generate the RNP complex. Electroporation Enhancer $(2 \mu \mathrm{L})$ (IDT; 1075916) and $2 \mu \mathrm{g}$ of plasmid were added to the RNP. During the annealing reaction, $1 \times 10^{6} \mathrm{BP}$ cells were pelleted at $600 \mathrm{xg}$ for $3 \mathrm{~min}$. The total volume of RNP + Plasmid + Electroporation Enhancer was transferred to an aspirated cell pellet. Next, $95 \mu \mathrm{L}$ of R buffer was added to the cells and they were gently resuspended to single cells for an immediate $100 \mu \mathrm{L}$ electroporation reaction. The Neon settings for the BP cells were two $30 \mathrm{~ms}$ pulses at 1150 volts. The cells were transferred to a single well of a 6 well plate and allowed to recover in growth media. After $24 \mathrm{hrs}$, the media was replaced and $1.5 \mu \mathrm{g} / \mu \mathrm{L}$ of Puromycin for a 48 -hr selection. The cells were allowed to recover from selection for $48 \mathrm{hrs}$ before singlecell clone selection. After clones were selected and expanded, AR protein was analyzed by Western Blot to confirm AR knockout. Briefly, cells were lysed for 30 min at $4{ }^{\circ} \mathrm{C}$ in $150 \mu \mathrm{L}$ RIPA buffer with phosphatase and protease inhibitors. Protein $(15 \mu \mathrm{g})$ was run on $10 \%$ SDS Page (Bio-Rad) and transferred overnight onto PDF membranes using TRIS Glycine Methanol buffer. After blocking for 1 hour at room temperature in 5\% milk diluted in TSBS-T, AR antibody (Abcam) was incubated at 1:2000 dilution for $24 \mathrm{hrs}$. GAPDH was used as a loading control. The lentiviral expression vectors pLV-105 were purchased from Genecopeia to express either GFP as a transduction control or mouse Ar (NM_013476.3) for an overexpression control. Lentivirus was generated using standard protocols and psPAX2 and pMD2.G as the packaging vectors. BP cells were transduced at $90 \%$ efficiency with viral supernatant and selected for 48 hours with $2 \mu \mathrm{g} / \mathrm{mL}$ puromycin.

\section{Measuring testosterone levels from plasma:}

Testosterone quantification was determined using Agilent's UHPLC Infinity II and 6495 triple quadrupole mass spectrometer, and MassHunter workstation software (8.0.8.23.5). Briefly, plasma samples were extracted with tert-butyl methyl ether (Sigma 34875), dried, and derivatized using hydroxylamine hydrochloride (Sigma 431362). The recovered ketoxime steroids were reconstituted in methanol/water $(1: 1 \mathrm{v} / \mathrm{v})$ and injected into the Infinity II UHPLC. Ketoxime steroids were separated using a Chromolith reverse phase column (RP-18 endcapped 100-2mm, Sigma 152006) and introduced into a JetStream source (Agilent) for triple quadrupole analysis. Data were analyzed and quantified using MassHunter software (Agilent) and GraphPad PRISM 8 software was used to graph and perform statistics (two-sided Students t-test) ${ }^{9,10}$.

\section{Immunofluorescence}


FFPE blocks were sectioned ( $5 \mu \mathrm{m})$, mounted on charged microscope slides (Leica 38002092), and dried at $37^{\circ} \mathrm{C}$ overnight. Slides were then baked at $60^{\circ} \mathrm{C}$ for 1 hour in an oven (Biocare DRY2008US), deparaffinized in 3 changes of xylene, and then rehydrated in 3 changes of $100 \%$ ethanol followed by a series of $95 \%, 70 \%$, and $50 \%$ ethanol and distilled water (5 min. each). Antigen retrieval (10 mM sodium citrate, $\mathrm{pH} 6.0$ with $0.05 \%$ Tween 20) was performed by heating slides to $95^{\circ} \mathrm{C}$ for 15 minutes in a microwave (Biogenex EZ Retriever System v.3) followed by a 30 minute cool down at room temperature. Slides were then washed with TBST (Thermo TA-999-TT). The area around each section was traced with a PAP pen (Sigma Aldrich Z672548), blocked with Background Sniper (Biocare BS966) for 10 minutes, and then washed with TBST.

Primary antibody (rabbit anti-Androgen Receptor [EPR1535(2)], Abcam ab133273, 1:300, diluted in Dako S3022) was incubated overnight at $4^{\circ} \mathrm{C}$, followed by a TBST wash. Secondary antibody (goat anti-rabbit Alexa-647 conjugate, Thermo A32733, 1:400, diluted in Fluorescence antibody Diluent, "FAD", Biocare FAD901L) was added and allowed to incubate for 1 hour at room temperature, followed by a TBST wash. A sequential incubation with fluorophore-conjugated primary antibody was performed (rabbit anti-Sodium Potassium ATPase [EP1845Y] Alexa-488 conjugate, Abcam ab197713, 1:500, were diluted in FAD) for 1 hour at room temperature, followed by a TBST wash. Finally, slides were incubated with DAPI $\left(4^{\prime}, 6-\right.$ Diamidino-2-Phenylindole, Dihydrochloride ( $5 \mathrm{mg} / \mathrm{mL}$ stock in DMF, Thermo D1306) at $0.25 \mu \mathrm{g} / \mathrm{mL}$ (diluted in TBST) for 10 minutes at room temperature. Slides were then washed with a series of TBST, followed by TBS and then distilled $\mathrm{H}_{2} \mathrm{O}$. Excess liquid was removed and slides were mounted with ProLong Diamond Gold (Life Tech P36930) and allowed to harden overnight. For patient samples, a TSAamplification of AR signal was performed. Briefly, following antigen retrieval/tissue tracing with PAP pen, performed as above, slides were incubated with treated with Bloxall to remove endogenous peroxidase (Vector Labs SP-6000) for 10 minutes at RT. Slides were then rinsed in water (30 seconds) and TBST (30 seconds). Slides were blocked with 2.5\% Normal Horse Serum (Vector Labs MP-7401 Component) for 25 minutes at RT and then block was tapped off. Slides were then blocked with Opal-specific PE/Diluent/Block (AKOYA ARD1001EA) for 10 minutes at RT and then block was tapped off. Primary antibody (rabbit anti-Androgen Receptor [EPR1535(2)], Abcam ab133273, 1:300, diluted in PE Diluent AKOYA ARD1001EA) was incubated $\mathrm{O} / \mathrm{N}$ at $4^{\circ} \mathrm{C}$, followed by a TBST wash for 3 minutes. Anti-Rabbit secondary HRP polymer was then added to slides (Impress MP-7401) for 25 minutes at RT after which slides were washed in TBST. Opal signal was generated by adding Opal 570 fluorophore (AKOYA FP1488001KT diluted 1:200 in 1x Plus Amplification Diluent AKOYA FP1609) for 10 minutes at RT and then washed in TBST. All following steps e.g. staining with the membrane marker (Abcam ab197713), DAPI, and coverslip mounting were identical to the steps described above.

\section{IF Image acquisition and Analysis}

Slides were imaged on a Vectra 3 using the A UPlanSApo 10x/0.40 air objective first. Images were acquired using all available channels with the Vectra software (3.0.5) and the raw data was saved as 
".qptiff" files. Regions of interest (ROIs) were created using PhenoChart (1.0.10) and these areas were then imaged again at 20X magnification on the Vectra. 20x images were then spectrally unmixed using inForm software and saved as Component TIFFs. The patient samples were imaged on a Vectra Polaris at 10x and saved as ".qptiff" files. Files were opened in QuPath software, channels were split, and saved individually (or merged) as TIFFs. An APP was created in Visiopharm to segment cells and assess intensity of AR immunofluorescence. Percent positive cells was calculated for each sample. Raw analyzed data was exported as a.CSV file and graphed/statistics run using GraphPad PRISM 8 software. A t-test test was performed to test significance.

\section{Methods References}

1 Amaria, R. N. et al. Neoadjuvant plus adjuvant dabrafenib and trametinib versus standard of care in patients with high-risk, surgically resectable melanoma: a single-centre, open-label, randomised, phase 2 trial. Lancet Oncol 19, 181-193, doi:10.1016/S1470-2045(18)30015-9 (2018).

2 FastQC: A Quality Control Tool for High Throughput Sequence Data [Online] (2010).

3 Dobin, A. et al. STAR: ultrafast universal RNA-seq aligner. Bioinformatics 29, 15-21, doi:10.1093/bioinformatics/bts635 (2013).

4 DeLuca, D. S. et al. RNA-SeQC: RNA-seq metrics for quality control and process optimization. Bioinformatics 28, 1530-1532, doi:10.1093/bioinformatics/bts196 (2012).

5 Anders, S., Pyl, P. T. \& Huber, W. HTSeq-a Python framework to work with high-throughput sequencing data. Bioinformatics 31, 166-169, doi:10.1093/bioinformatics/btu638 (2015).

6 Love, M. I., Huber, W. \& Anders, S. Moderated estimation of fold change and dispersion for RNA-seq data with DESeq2. Genome Biol 15, 550, doi:10.1186/s13059-014-0550-8 (2014).

7 Huber, W., von Heydebreck, A., Sultmann, H., Poustka, A. \& Vingron, M. Variance stabilization applied to microarray data calibration and to the quantification of differential expression. Bioinformatics 18 Suppl 1, S96-104, doi:10.1093/bioinformatics/18.suppl_1.s96 (2002).

8 Tibes, R. et al. Reverse phase protein array: validation of a novel proteomic technology and utility for analysis of primary leukemia specimens and hematopoietic stem cells. Mol Cancer Ther 5, 2512-2521, doi:10.1158/1535-7163.MCT-06-0334 (2006).

9 Efstathiou, E. et al. Enzalutamide in Combination with Abiraterone Acetate in Bone Metastatic Castration-resistant Prostate Cancer Patients. Eur Urol Onco/ 3, 119-127, doi:10.1016/j.euo.2019.01.008 (2020).

10 Maity, S. N. et al. Targeting of CYP17A1 Lyase by VT-464 Inhibits Adrenal and Intratumoral Androgen Biosynthesis and Tumor Growth of Castration Resistant Prostate Cancer. Sci Rep 6, 35354, doi:10.1038/srep35354 (2016). 


\section{Declarations}

\section{ACKNOWLEDGEMENTS}

JAW is supported by the NIH (1 R01 CA219896-01A1), U.S- Israel Binational Science Foundation (201332), the Melanoma Research Alliance (4022024), American Association for Cancer Research Stand Up To Cancer (SU2C-AACR-IRG-19-17), Department of Defense (W81XWH-16-1-0121), MD Anderson Cancer Center Multidisciplinary Research Program Grant, Andrew Sabin Family Fellows Program, and MD Anderson Cancer Center's Melanoma Moon Shots Program. JAW is a member of the Parker Institute for Cancer Immunotherapy at MD Anderson Cancer Center.

JLM is supported by the Melanoma Research Alliance, an American Society of Clinical Oncology and Conquer Cancer Foundation Career Development Award, the Elkins Foundation, Seerave Foundation, Rising Tide Foundation, the Mark Foundation, an MD Anderson Cancer Center (MDACC) Melanoma SPORE Developmental Research Program Award, and the MD Anderson Physician Scientist Program and acknowledges the Transdisciplinary Research in Energetics and Cancer Research Training Workshop R25CA203650 and the MDACC Center for Energy Balance in Cancer Prevention and Survivorship.

APC is supported by the CPRIT Research Training Program at MD Anderson Cancer Center (RP170067), The U.S. Department of State, Bureau of Educational and Cultural Affairs, and the Fulbright FrancoAméricaine Commission.

MCA is supported by a National Health and Medical Research Council of Australia CJ Martin Early Career Fellowship (\#1148680).

BH was supported by National Institutes of Health T32 CA 009599 and the MD Anderson Cancer Center support grant (P30 CA016672).

MGW is supported by National Institutes of Health T32 CA 009599 and the MD Anderson Cancer Center support grant (P30 CA016672).

MAD is supported by NIH/NCI (2T32CA009666-21), the Dr. Miriam and Sheldon G. Adelson Medical Research Foundation, AIM at Melanoma Foundation and philanthropic contributions to the Melanoma Moon Shots Program of MD Anderson Cancer Center.

GOO is supported by National Institutes of Health T32 CA 009599 and the MD Anderson Cancer Center support grant (P30 CA016672).

\section{AUTHOR CONTRIBUTIONS}

CPV, MGW, MCA, JW - formulated research goals and aims 
CPV, MGW, MCA, JW - developed and designed methodology

MGW, MCA, HZ - designed and executed pipelines and analytic code

CPB, MGW, MCA, HZ, GM - conducted statistical analysis, built models and plotted results CPV, JRM, JM

- designed and conducted immunological assays and pre-clinical studies generated and annotated meta data, created cell lines

JK, SJ, NF, GG- in vitro studies

CPV, JRM - scientific drivers, planned and oversaw all murine experiments and studies

JRD, MM - AR staining

MM - RPPA and quant-seq data

MP - generation of AR knockout cell lines

LF, JRD, JM, JW,prepared the manuscript

TH - Scientific oversight

All authors reviewed and edited the manuscript

JW - supervised and oversaw all aspects of study including design, conduct and analyses

\section{DISCLOSURE}

J. Wargo is an inventor on a US patent application (PCT/US17/53.717) submitted by the University of Texas MD Anderson Cancer Center that covers methods to enhance immune checkpoint blockade responses by modulating the microbiome. J. Wargo reports compensation for speaker's bureau and honoraria from Imedex, Dava Oncology, Omniprex, Illumina, Gilead, PeerView, Physician Education Resource, Medlmmune and Bristol-Myers Squibb. J. Wargo serves as a consultant / advisory board member for Roche/Genentech, Novartis, AstraZeneca, GlaxoSmithKline, Bristol-Myers Squibb, Merck, Biothera Pharmaceuticals and Microbiome DX. J. Wargo also receives research support from GlaxoSmithKline, Roche/Genentech, Bristol-Myers Squibb, and Novartis.

CNS is a contributor on a US patent application (PCT/US17/53.717) submitted by the University of Texas MD Anderson Cancer Center that covers methods to enhance immune checkpoint blockade responses by modulating the microbiome.

VG is a co-inventor on a US patent application (PCT/US17/53 717) submitted by The University of Texas MD Anderson Cancer Center that covers methods to enhance checkpoint blockade therapy by the microbiome. V.G. has served as a consultant for Microbiome DX, and reports honoraria from ExpertConnect and Kansas Society of Clinical Oncology. 
JLM serves as a consultant for Merck and in advisory board for Bristol-Myers Squibb.

MCA reports advisory board participation and honoraria from Merck Sharp and Dohme, outside the submitted work.

RNA receives research funding from Merck, Bristol-Myers Squibb, Genentech, Novartis, lovance and is a consult for lovance and Nektar.

MAD is an advisory board member/consultant for BMS, Novartis, Array, Roche/Genentech, GSK SanofiAventis. PI of research grant to institution from Astrazeneca, Roche/Genentech, GSK, Myriad, Oncothyreon, Sanofi-Aventis.

\section{All other authors report no COI directly relevant to this work.}

\section{MATERIALS AND CORRESPONDENCE}

Further information and requests for resources and reagents should be directed to and will be fulfilled by the Lead Contacts, Timothy Heffernan (TPHeffernan@mdanderson.org), Joseph Marszalek (JRMarszalek@mdanderson.org), or Jennifer A.Wargo (jwargo@mdanderson.org).

\section{References}

1 Robert, C. et al. Five-Year Outcomes with Dabrafenib plus Trametinib in Metastatic Melanoma. The New England journal of medicine 381, 626-636, doi:10.1056/NEJMoa1904059 (2019).

2 Conforti, F. et al. Cancer immunotherapy efficacy and patients' sex: a systematic review and metaanalysis. Lancet Oncol 19, 737-746, doi:10.1016/S1470-2045(18)30261-4 (2018).

3 Long, G. V. et al. Factors predictive of response, disease progression, and overall survival after dabrafenib and trametinib combination treatment: a pooled analysis of individual patient data from randomised trials. Lancet Oncol 17, 1743-1754, doi:10.1016/S1470-2045(16)30578-2 (2016).

$4 \quad$ Ma, J., Malladi, S. \& Beck, A. H. Systematic Analysis of Sex-Linked Molecular Alterations and Therapies in Cancer. Sci Rep 6, 19119, doi:10.1038/srep19119 (2016).

5 Clocchiatti, A., Cora, E., Zhang, Y. \& Dotto, G. P. Sexual dimorphism in cancer. Nat Rev Cancer 16, 330-339, doi:10.1038/nrc.2016.30 (2016).

6 Miyamoto, H. et al. Promotion of bladder cancer development and progression by androgen receptor signals. J Natl Cancer Inst 99, 558-568, doi:10.1093/jnci/djk113 (2007).

7 Mizushima, T. \& Miyamoto, H. The Role of Androgen Receptor Signaling in Ovarian Cancer. Cells 8, doi:10.3390/cells8020176 (2019). 

colorectal cancer. Clin Cancer Res 19, 5842-5848, doi:10.1158/1078-0432.CCR-13-0325 (2013).

9 Pinto, J. A. et al. Gender and outcomes in non-small cell lung cancer: an old prognostic variable comes back for targeted therapy and immunotherapy? ESMO Open 3, e000344, doi:10.1136/esmoopen2018-000344 (2018).

10 Capone, I., Marchetti, P., Ascierto, P. A., Malorni, W. \& Gabriele, L. Sexual Dimorphism of Immune Responses: A New Perspective in Cancer Immunotherapy. Front Immuno/ 9, 552, doi:10.3389/fimmu.2018.00552 (2018).

11 Clocchiatti, A. et al. Androgen receptor functions as transcriptional repressor of cancer-associated fibroblast activation. J Clin Invest 128, 5531-5548, doi:10.1172/JCI99159 (2018).

12 Pequeux, C. et al. Stromal estrogen receptor-alpha promotes tumor growth by normalizing an increased angiogenesis. Cancer Res 72, 3010-3019, doi:10.1158/0008-5472.CAN-11-3768 (2012).

13 Zhao, L. et al. Pharmacological activation of estrogen receptor beta augments innate immunity to suppress cancer metastasis. Proc Natl Acad Sci U S A 115, E3673-E3681, doi:10.1073/pnas.1803291115 (2018).

14 Rothenberger, N. J., Somasundaram, A. \& Stabile, L. P. The Role of the Estrogen Pathway in the Tumor Microenvironment. Int J Mol Sci 19, doi:10.3390/ijms19020611 (2018).

15 Hoang, D. T., Iczkowski, K. A., Kilari, D., See, W. \& Nevalainen, M. T. Androgen receptor-dependent and -independent mechanisms driving prostate cancer progression: Opportunities for therapeutic targeting from multiple angles. Oncotarget 8, 3724-3745, doi:10.18632/oncotarget.12554 (2017).

16 Ribeiro, M. P. C., Santos, A. E. \& Custodio, J. B. A. The activation of the G protein-coupled estrogen receptor (GPER) inhibits the proliferation of mouse melanoma K1735-M2 cells. Chem Biol Interact 277, 176-184, doi:10.1016/j.cbi.2017.09.017 (2017).

17 Qi, J. Therapy resistance by splicing: can the androgen receptor teach us about BRAF? Pigment Cell Melanoma Res 25, 293-294, doi:10.1111/j.1755-148x.2012.01000.x (2012).

18 Wang, Y. et al. Androgen receptor promotes melanoma metastasis via altering the miRNA-5393p/USP13/MITF/AXL signals. Oncogene 36, 1644-1654, doi:10.1038/onc.2016.330 (2017).

19 Natale, C. A. et al. Activation of G protein-coupled estrogen receptor signaling inhibits melanoma and improves response to immune checkpoint blockade. Elife 7, doi:10.7554/eLife.31770 (2018).

20 Smalley, K. S. Why do women with melanoma do better than men? Elife 7, doi:10.7554/eLife.33511 (2018). 
21 Marzagalli, M. et al. Estrogen Receptor beta in Melanoma: From Molecular Insights to Potential Clinical Utility. Front Endocrinol (Lausanne) 7, 140, doi:10.3389/fendo.2016.00140 (2016).

22 Amaria, R. N. et al. Neoadjuvant plus adjuvant dabrafenib and trametinib versus standard of care in patients with high-risk, surgically resectable melanoma: a single-centre, open-label, randomised, phase 2 trial. Lancet Onco/ 19, 181-193, doi:10.1016/S1470-2045(18)30015-9 (2018).

23 Long, G. V. et al. Neoadjuvant dabrafenib combined with trametinib for resectable, stage IIIB-C, BRAF(V600) mutation-positive melanoma (NeoCombi): a single-arm, open-label, single-centre, phase 2 trial. Lancet Oncol 20, 961-971, doi:10.1016/S1470-2045(19)30331-6 (2019).

24 Lewis, K. D. et al. Impact of depth of response on survival in patients treated with cobimetinib +/vemurafenib: pooled analysis of BRIM-2, BRIM-3, BRIM-7 and coBRIM. Br J Cancer 121, 522-528, doi:10.1038/s41416-019-0546-y (2019).

25 Andrews MC, C. S., Hudgens CW, Tetzlaff MT, Amaria RN, Arur S, Heffernan TP, Wargo JA. in Society for Melanoma Research (SMR) (Manchester, England, 2018).

26 Amaria, R. N. et al. Neoadjuvant systemic therapy in melanoma: recommendations of the International Neoadjuvant Melanoma Consortium. Lancet Oncol 20, e378-e389, doi:10.1016/S14702045(19)30332-8 (2019).

\section{Figures}


A

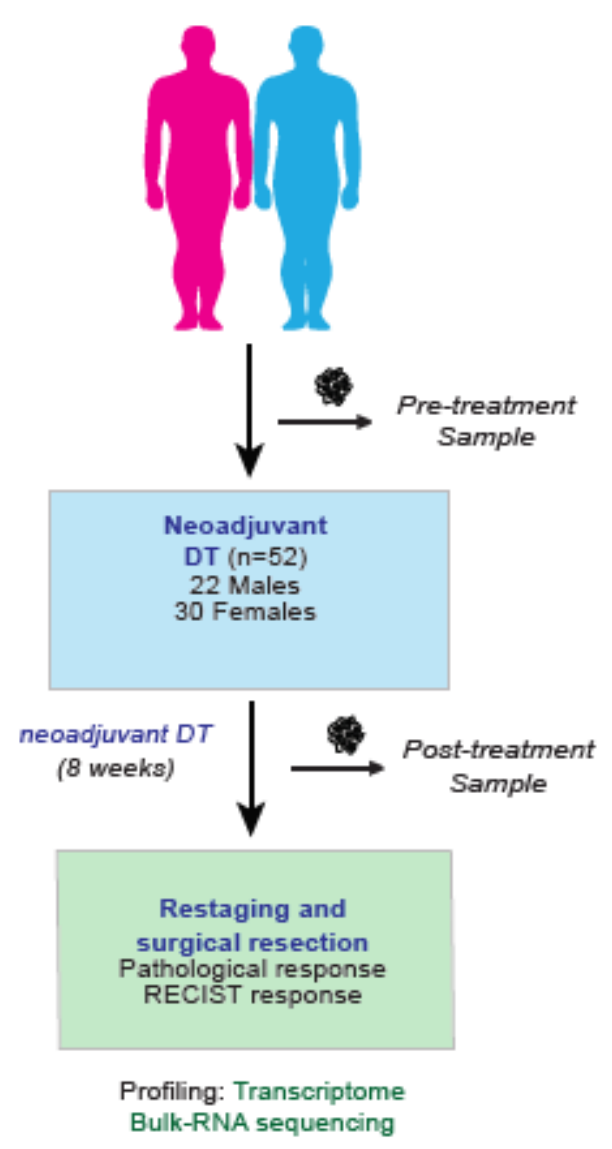

B

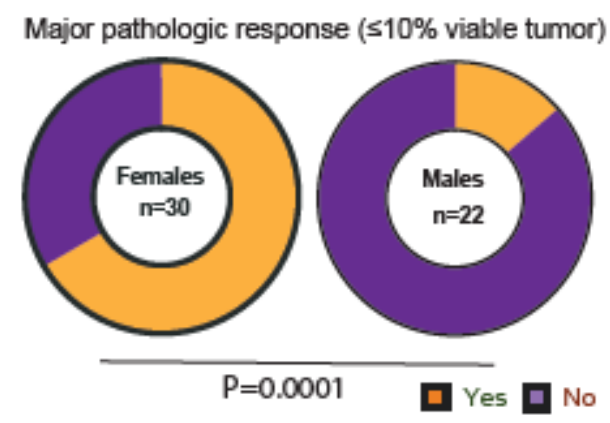

C

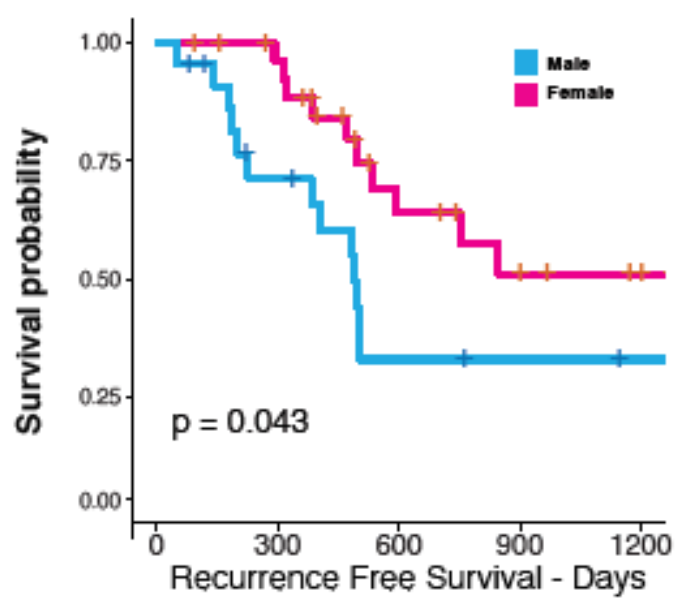

D

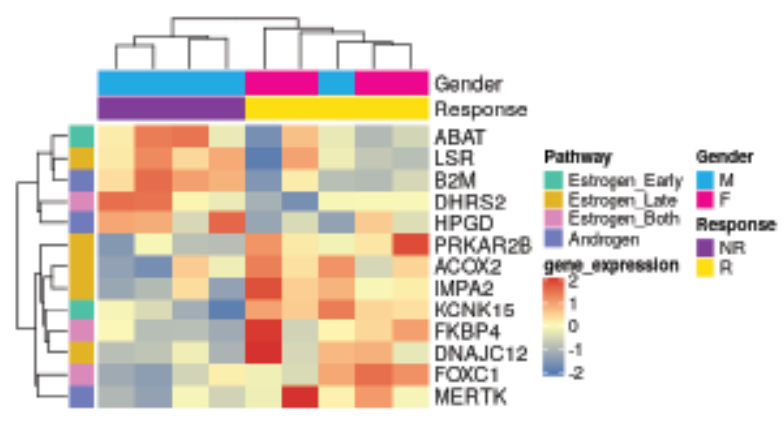

E

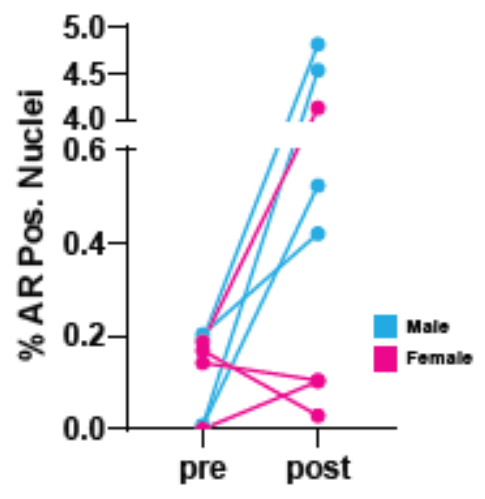

Figure 1

Strong sexual dimorphism noted in response to neoadjuvant BRAF-MEK inhibition therapy in melanoma patients Sex of the patient influences response to targeted therapy in melanoma, A) Schema of Clinical cohort of patients treated with targeted therapy, B) Major pathologic response with $\leq 10 \%$ viable tumor in females and males, C) Recurrence-free survival by sex, D) Differentially expressed genes in pre-treatment specimens between responders and non-responders in the hallmark estrogen and androgen response pathways, E) Androgen receptor staining pre- and post-treatment in males (blue) and females (pink). 
A

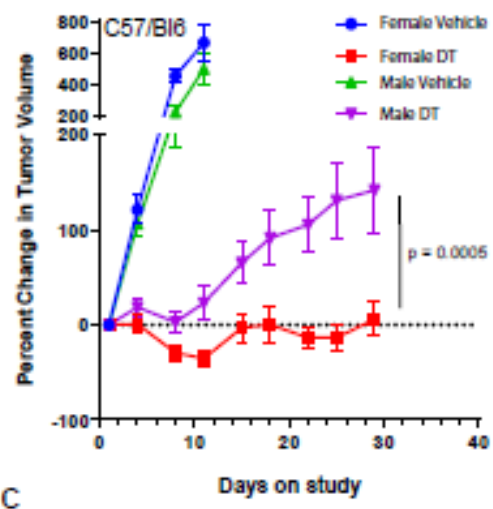

$\mathrm{C}$

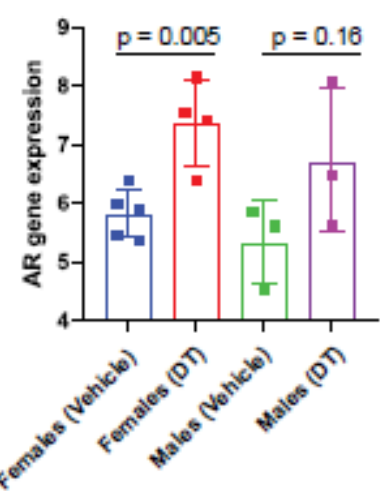

E

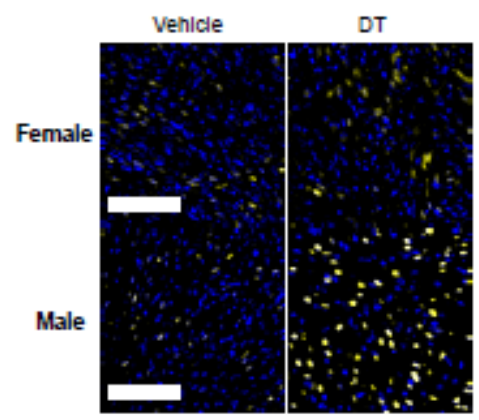

B

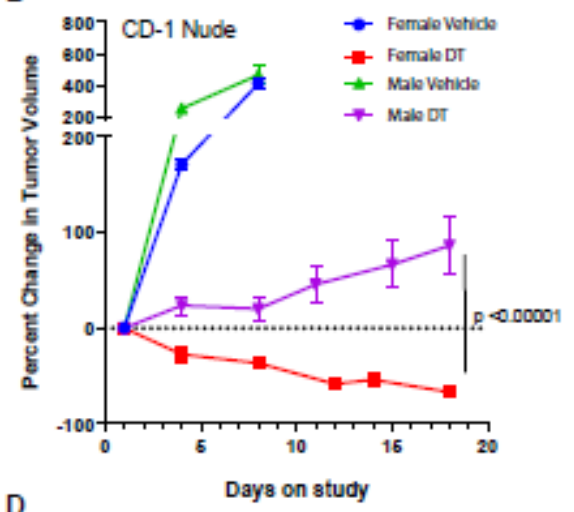

D
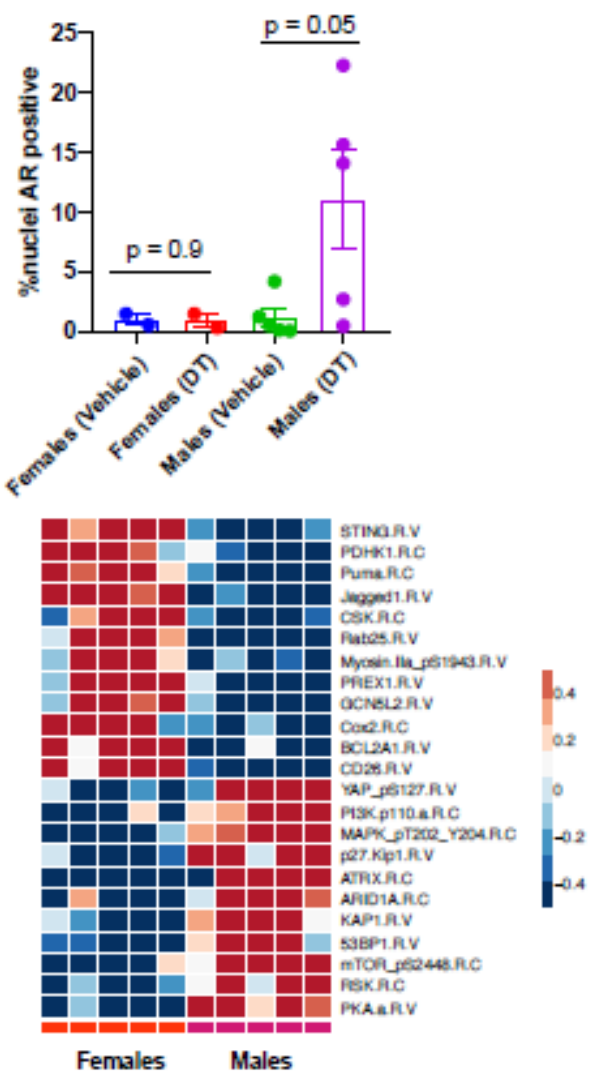

Figure 2

Murine model of melanoma validates a sexual dimorphic response and suggests AR activity as a mechanism of resistance A) Percent change in tumor volume for male and female C57BI/6 mice implanted subcutaneously with BP (BRAFV600E, PTEN-/-) cells that were treated with Vehicle or DT (30 mpk Dabrafenib and 1 mpk Trametinib, PO, QD). $n=10$ mice per group, and the experiment was performed three times (see Supplementary Figure 3B-C). B) Percent change in tumor volume for male and female CD-1 nude mice implanted and treated as in $A . n=10$ mice per group. For $A$ and $B, p$-values were calculated for the final time point using two-sided Student's t-test. C) Transcriptome based mRNA expression of Androgen Receptor (AR) from Quant-seq data in female and male mice treated with Vehicle and DT. p-values were calculated using two-sided Student's t-test. D) Percentage of cells with AR positive nuclei in BP tumors from male and female BP mice treated with vehicle or DT for 25 days. p-values were calculated using two-sided Student's t-test. E) Representative images of AR protein expression by 
immunofluorescence across the four groups of mice in D. Blue = DAPI; Yellow = Androgen Receptor. Scale $=100 \mu \mathrm{m}$. F) Differential protein expression for several proteins measured using RPPA. p-values comparing vehicle to treated samples were calculated using two-sided Student's t-test and proteins with $\mathrm{p}<0.009$ are shown.
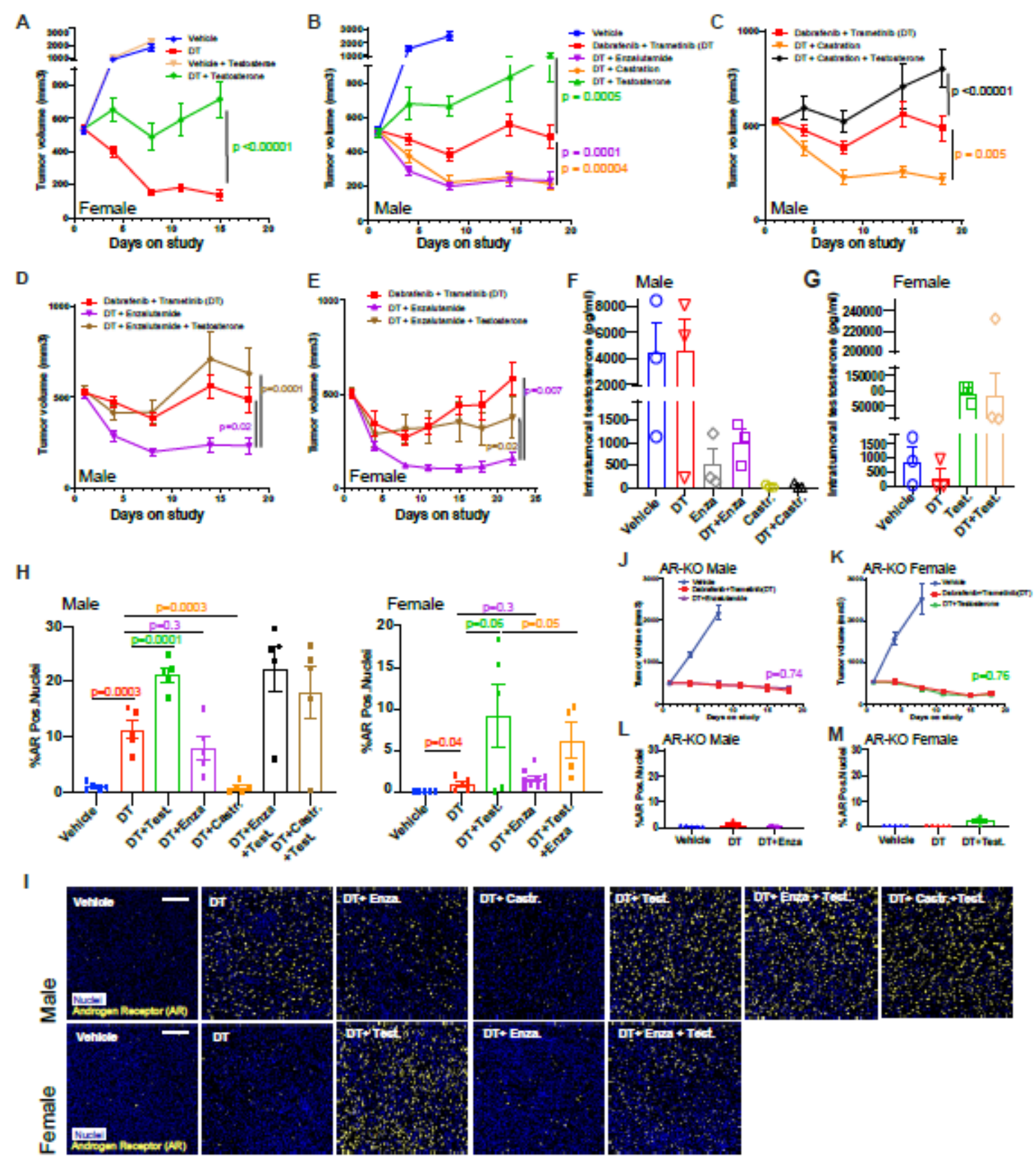

\section{Figure 3}

Modulation of AR activity is associated with differential response to DT A) Percent change in BP tumor growth for female mice treated with vehicle, DT, or DT in combination with testosterone, and B) male mice treated with vehicle, DT, or DT in combination with enzalutamide, physical castration, or testosterone. C) Percent change in BP tumor growth in male mice treated with DT alone or DT in combination with physical castration or castration plus testosterone supplementation. D) Male and E) female mice treated 
with DT alone or DT in combination with either enzalutamide or enzalutamide plus testosterone supplementation. ( $n=6-10$ mice/group). For A-E, $p$-values were calculated for the final time point using two-sided Student's t-test. Note that the DT, DT + Castration, and DT + Enzalutamide groups in C and D are the same data that is shown in $B$. F and $G$ ) Level of testosterone $(\mathrm{pg} / \mathrm{mL})$ in plasma samples from BP tumor growth experiments in male and female mice, respectively. p-value was calculated using two-sided Student's t-test. H) Quantification of the percent of AR+ nuclei by immunofluorescence in BP tumors from male and female mice treated for 25 days. p-values were calculated using two-sided Student's t-test. I) Representative images from samples in H. Blue = DAPl; Yellow $=$ Androgen Receptor. Scale $=100 \mu \mathrm{m} . \mathrm{J}-$ K) Percent change in AR-KO BP tumor growth for female $(\mathrm{J})$ and male $(\mathrm{K})$ mice treated with vehicle or DT in combination with either enzalutamide or testosterone, respectively ( $\mathrm{n}=10 \mathrm{mice} / \mathrm{group})$.

\section{Supplementary Files}

This is a list of supplementary files associated with this preprint. Click to download.

- Suppfig1.pdf

- supplement2.pdf

- supplement3.pdf

- SupplementalTable1.xIsx

- SupplementalTable2.xIsx 